\title{
Special issue on intelligent urban computing with big data
}

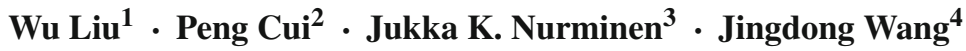

Published online: 12 September 2017

(C) Springer-Verlag GmbH Germany 2017

The rapid proliferation of urbanization has modernized many people's lives and also engendered critical issues, such as traffic congestion, energy consumption, and environmental pollution. These urbanization challenges seriously deteriorate people's life quality in big cities. Nowadays, sensing technologies and large-scale computing infrastructures have produced a variety of big data in urban spaces, which provide rich knowledge about a city to help tackle these challenges. Consequently, the intelligent urban computing, which holistically exploits the big data in big cities to improve the urban environment, human life quality, and city operation systems, has obtained massive attention in research and industrial fields. Many efforts have been dedicated to connecting unobtrusive and ubiquitous sensing technologies, advanced data management and analytics models, and novel visualization methods to structure intelligent urban computing systems for smart cities. Furthermore, the intelligent systems and applications are emerging and becoming pervasive in the

\section{Wu Liu \\ liuwu@bupt.edu.cn \\ Peng Cui \\ cuip@tsinghua.edu.cn \\ Jukka K. Nurminen \\ jukka.k.nurminen@vtt.fi \\ Jingdong Wang \\ jingdw@microsoft.com}

1 Beijing Key Lab of Intelligent Telecommunication Software and Multimedia, Beijing University of Posts and Telecommunications, Beijing 100876, China

2 Lab of Media and Network, Department of Computer Science and Technology, Tsinghua University, Beijing 100084, China

3 VTT Technical Research Centre of Finland, PO Box 1000, 02044 VTT, Espoo, Finland

4 Microsoft Research Asia, Beijing 100080, China field of urban planning, transportation systems, environmental conservation, energy consumption, social applications, economy, public security, and presenting representative scenarios.

The goal of this special issue is to call for a coordinated effort to understand the opportunities and challenges emerging in intelligent urban computing with big data, identify key tasks, and evaluate the state-of-the-art methods, showcase innovative methodologies and ideas, introduce interesting real-world intelligent urban computing systems or applications, propose new real-world datasets and discuss future directions. We solicit original contributions in all fields of intelligent urban computing that explore the big data in big cities to help us understand the nature of urban phenomena and even predict the future of cities.

With the assistance of professional referees, 11 papers out from 33 submissions are accepted after at least two rounds of rigorous reviews. Four of them were developed from conference contributions at IEEE CCIS 2016 [4,5,7,10]. Seven other papers are selected in response to an open call for papers. These papers cover a wide range of subtopics of intelligent urban computing with big data, including intelligent video surveillance $[4,5,11]$, intelligent urban sensing technologies $[8,10]$, machine vision algorithms in urban computing [2,3,9], intelligent traffic system [7], 3D vision in urban computing $[1,6]$.

In the first paper, Huang et al. [4] propose a novel human body segmentation framework based on shape constraint. The shape constraint is the combination of human star convexity and body parts' locations with high precision. The experimental results demonstrate that the proposed method outperforms many state-of-the-art methods on public challenging datasets.

Li et al. [5] propose a new person re-identification framework. They firstly design a Siamese Inception network to 
extract the high-level semantic features, which are robust to the human appearance changes in different camera views. Then the semantic features are combined with the low-level features (e.g., HOG, LOMO, Color Name) through the Null Foley-Sammon Transform-based metric learning approach. The comprehensive evaluations demonstrate that the proposed framework achieves the best performance on four widely used person re-identification benchmarks.

Zhuo et al. [11] propose a vehicle classification dataset named "VehicleDataset," which contains 13,700 images extracted from real surveillance videos in various changes of resolutions, illumination, noise, angle of video cameras, and weather. The vehicles are classified into six categories, i.e., bus, car, motorcycle, minibus, truck, and van. They also evaluate different baseline methods on the dataset. Among them, the fine-tuned GoogLeNet model achieves the best performance.

Song et al. [8] propose a robust and parallel Uyghur text localization method in complex background images. The method firstly employs a multicolor-channel enhanced maximally stable extremal regions to extract components in images, which is robust to blur, low resolution, and low contraction. Then a two-stage component classifier is exploited to precisely distinguish texts from a large number of non-test components. Moreover, a component-connected graph algorithm is proposed to construct text lines. Finally, an effective heterogeneous parallelization scheme is proposed to achieve 12.5 times speedup.

Zhao et al. [10] propose a mobile indoor shop recognition system via user-friendly and efficient fingerprinting on smartphones. The fingerprinting method collects various valuable sensory data, including Wi-Fi RSS, azimuth angle, tilt angle, shop image, and utilizes an efficient fingerprint searching and matching method to improve the recognition accuracy. Moreover, they also release two fingerprint datasets, which are collected by the proposed prototype system.

Chen et al. [2] solve the graph-matching problem in machine vision based on the typical graduated non-convexity and concavity procedure (GNCCP) algorithm. To address the singularity of points in the solution path gained by the GNCCP, they firstly propose a singular points discovering method by checking smoothness of the path. Then multiple paths are explored at singular points by the adaptive path estimation. Finally, the best one is selected in line with objectives from all the balanced paths. The proposed algorithm achieves better objectives than GNCCP.

Huang et al. [3] survey several memory deduplication techniques for intelligent urban computing. First of all, limited memory size is considered as a major bottleneck in data centers for intelligent urban computing. Memory deduplication aims to automatically eliminate duplicate data in memory. This paper classifies the memory deduplication techniques into five categories and highlights their similari- ties and differences. It will provide insights to researchers and motivate them to deploy intelligent urban computing with a realizable memory requirement.

$\mathrm{Xu}$ et al. [9] design a binary embedding framework to retrieve relevant images from the large database. Different from the existed methods quantize each projected dimension to one bit, they propose a novel triple-bit quantization strategy to assign 3-bit to each dimension. Then, the asymmetric distance algorithm is applied to re-rank the searched candidates in the Hamming space. The evaluations demonstrate that the proposed method can achieve remarkable improvement in query accuracy compared to original binary embedding methods.

Shen et al. [7] improve the weighted extreme learning machine (ELM) with decay-weight matrix setting. By optimal selection of decaying coefficient, the weighted ELM can adjust the weight sum value of each class and then adjust class boundary. This makes the method obtain better effects in solving the imbalance classification tasks, particularly in multi-class tasks. This method is successfully applied to build the prediction model in urban traffic congestion prediction system.

Lv et al. [6] propose a 3D laser scanning technology based on the pin-hole camera with lens distortion to restore the 3D data of the measured object. With the stepper motor and camera, the authors design the professional 2D RAC calibration, $3 \mathrm{D}$ reconstruction, and 3D volume calculation to obtain the object 3D surface information.

In the last paper, Babahajiani et al. [1] present a multiple stage methodology for urban 3D segmentation. The method firstly takes GPS registered Light Detection And Ranging (LiDAR) point clouds and street view images as inputs. Then with the two inputs, the semantic labels are annotated for the 3D LiDAR points clouds through a combination of fast rule-based parsing and boosted decision trees. Experimental results on three popular datasets demonstrate the superior accuracy and efficiency of the proposed method.

The guest editors would like to thank the authors for their efforts and interest. Moreover, we would also like to state how much we appreciate the valuable comments and suggestions provided by the reviewers. Lastly, a special thanks to the Editor-in-Chief Prof. Rahul Sukthankar and to the Editorial Coordinator Cherry Place for their support during the preparation and publication of this special issue.

\section{References}

1. Babahajiani, P., Fan, L., Kämäräinen, J.K., Gabbouj, M.: Urban $3 \mathrm{~d}$ segmentation and modelling from street view images and LiDAR point clouds. Mach. Vis. Appl. (2017). doi:10.1007/ s00138-017-0845-3 
2. Chen, R., Lang, C., Wang, T.: Multiple path exploration for graph matching. Mach. Vis. Appl. (2017). doi:10.1007/ s00138-017-0847-1

3. Huang, H., Yan, C., Liu, B., Chen, L.: A survey of memory deduplication approaches for intelligent urban computing. Mach. Vis. Appl. (2017). doi:10.1007/s00138-017-0834-6

4. Huang, L., Nie, J., Wei, Z.: Human body segmentation based on shape constraint. Mach. Vis. Appl. (2017). doi:10.1007/ s00138-017-0829-3

5. Li, S., Ma, H.: A Siamese inception architecture network for person re-identification. Mach. Vis. Appl. (2017). doi:10.1007/ s00138-017-0843-5

6. Lv, B., Li, L., Yan, C.: Three-dimensional laser scanning under the pinhole camera with lens distortion. Mach. Vis. Appl. (2017). doi:10.1007/s00138-017-0854-2

7. Shen, Q., Ban, X., Liu, R., Wang, Y.: Decay-weighted extreme learning machine for balance and optimization learning. Mach. Vis. Appl. (2017). doi:10.1007/s00138-017-0828-4

8. Song, Y., Chen, J., Xie, H., Chen, Z., Gao, X., Chen, X.: Robust and parallel Uyghur text localization in complex background images. Mach. Vis. Appl. (2017). doi:10.1007/s00138-017-0837-3

9. Xu, D., Xie, H., Yan, C.: Triple-bit quantization with asymmetric distance for image content security. Mach. Vis. Appl. (2017). doi:10.1007/s00138-017-0853-3

10. Zhao, D., Xu, H., An, J., Liu, L., Ma, H.: Isr: indoor shop recognition via user-friendly and efficient fingerprinting on smartphones. Mach. Vis. Appl. (2017). doi:10.1007/s00138-017-0838-2

11. Zhuo, L., Jiang, L., Zhu, Z., Li, J., Zhang, J., Long, H.: Vehicle classification for large-scale traffic surveillance videos using convolutional neural networks. Mach. Vis. Appl. (2017). doi:10.1007/ s00138-017-0846-2

Wu Liu is a Lecturer in Beijing University of Posts and Telecommunications, China. He received his Ph.D. degree from Institute of Computing Technology (ICT), Chinese Academy of Science (CAS) in 2015. From December 2014 to April 2015, he was a visiting student in the University of Rochester, NY, USA. His current research interests include multimedia analysis and retrieval, and computer vision. He has published more than 30 papers in prestigious conferences and journals in computer vision and multimedia. His recent research won the Chinese Academy of Sciences Outstanding Ph.D. Thesis Award in 2016, and Best Student Paper Awards at ICME in 2016.
Peng Cui is an assistant professor and leads the research in media and network lab in Tsinghua University. He is keen to promote the convergence of social media data mining and multimedia computing technologies, and received several paper awards in prestigious conferences on multimedia or data mining, including ACM MM, KDD, ICME, ICDM, MMM, in recent years. He is the AE of ACM TOMM, Neurocomputing, and area chairs of ACM Multimedia, ICME, ICDM, etc, and was awarded the ACM China Rising Star in 2015.

Jukka K. Nurminen is a principal scientist at VTT and an adjunct professor of computer science at Aalto University. In 2011-2015, he spent 5 years as a professor at Aalto working on mobile cloud computing and energy efficiency. He has a strong industry background with almost 25 years of experience in software research at Nokia Research Center. Jukka's experience ranges from mathematical modeling to expert systems, from network planning tools to solutions for mobile phones, and from $R \& D$ project management to tens of patented inventions. Jukka received his M.Sc. degree in 1986 and Ph.D. degree in 2003 from Helsinki University of Technology. His wide range of research interests are focused on systems and solution that are smart, energy efficient, distributed, or mobile.

Jingdong Wang is a Senior Researcher at the Visual Computing Group, Microsoft Research Asia. He received the B.Eng. and M.Eng. degrees from the Department of Automation, Tsinghua University, Beijing, China, in 2001 and 2004, respectively, and the PhD degree from the Department of Computer Science and Engineering, the Hong Kong University of Science and Technology, Hong Kong, in 2007. His areas of interest include deep learning, large-scale indexing, human understanding, and person re-identification. He has been serving/served as an Associate Editor of IEEE TMM, and an area chair of ICCV 2017, CVPR 2017, ECCV 2016, and ACM Multimedia 2015. 